\title{
Effects of ingredients and process conditions on 'Amaretti' cookies characteristics
}

\author{
Stefano Farris* \& Luciano Piergiovanni
}

diSTAM, Department of Food Science and Microbiology, University of Milan, via Celoria 2, 20133 - Milano, Italy

Summary This research aimed at determining the influence of different factors (i.e. ingredients and process conditions) on the most important characteristics defining consumers appreciation of 'Amaretti' cookies using Design Of Experiment (DOE) technique. A different recipe than the original one was used in the manufacturing of the cookies, where saccharose was partially replaced with fructose and bamboo fibre was added as a new ingredient. Besides fructose/saccharose ratio and fibre, the effect of egg white, baking time and baking temperature on quality responses (hardness, water activity, moisture content and colour) of 'Amaretti' was measured by using a fractional factorial design in a screening test. Responses were affected mostly by changes in temperature and fructose/saccharose ratio levels and then by baking time; bamboo fibre had a statistically significant influence only on hardness. The power of fit of the regression models was significant for all four responses and had $R^{2}$ value in the range of $0.886-0.997$. However, the power of prediction was significant only for hardness, moisture content and colour and had $Q^{2}$ value in the range of $0.584-0.965$. The mathematical model for water activity resulted inappropriate to explain the link between factors and response. The achieved results can represent an useful tool to address and facilitate the next phase of optimisation.

Keywords baking, cookies, ingredients, screening design, shelf-life.

\footnotetext{
*Correspondent: Fax: +39025031 6672;

e-mail: stefano.farris@unimi.it
} 


\section{Introduction}

'Amaretti' is the name of Italian food products well-known to a large population in the world; apart from the famous liqueur flavoured with herbs and fruits soaked in apricot kernel oil, this name is used for almond paste cookies found all over the country even if prepared in different ways. In fact, they are manufactured using different traditional formulations and processing but, despite the incorporation of technological progresses, their manufacturing remains in the line with methods used in the past (Trichopoulou et al., 2006). Due to this, their popularity is growing day by day also abroad as typical and traditional Italian cookies; however, some problems in the export of 'Amaretti' still exist and most of them are associated with the change of cookie texture after a few days that strictly limits their shelf-life. In fact, as reported in a previous work (Farris et al, 2006) they are characterized by a soft internal almond paste $\left(a_{w} \approx 0.74\right.$; moisture content $\left.\approx 5 \%\right)$ whereas their external crust is crunchy and dry $\left(\mathrm{a}_{\mathrm{w}} \approx 0.40\right.$; moisture content $\left.\approx 14 \%\right)$. Hence, the "Amaretto" is an example of a multi-domain system, that is, a food having two or more regions or components with different moisture content and $a_{w}$. This contrast in water activity $\left(a_{w}\right)$ implies a non-equilibrium thermodynamic state that lead to a qualitative decay manifested in a severe hardening of the internal almond paste due both to the redistribution of water (that yield to the sugar crystallization) and loss of water into the surrounding environment (Piga, et al., 2005). Therefore, the major problem is the subsequent loss in textural properties (differences between crust and paste) during shelf life that, along with colour and taste, is considered the most important quality attribute by consumers, as reported in published research papers (Farris et al., 2006; Guillard et al., 2003a,b; Labuza \& Hyman, 1998; Ramos-Cabrer et al., 2006; Roca et al., 2006). To solve this kind of problem, modifications on the original formula are recognized as a potential tool for the control of the overall quality attributes of existing foods (Manzocco \& Nicoli, 2002). So, two different ingredients were added to the original recipe of 'Amaretti': fructose, in partial substitution of saccharose, and bamboo fibre. Fructose was chosen as humectant and anti-crystallizing agent (Guilbért, 2002). Because of its colligative properties, it can be easily used to lower the water activity of different 
food systems, showing an high water binding capacity. Finally, achieving a lower water activity, leads to a greater microbial stability keeping the same moisture content into the food. The solubility of fructose in water at $25^{\circ} \mathrm{C}$ is $4 \mathrm{~g} \mathrm{~g} \mathrm{~g}^{-1} \mathrm{H}_{2} \mathrm{O}$, which is the highest value of most important sugars and sugar alcohols. Due to this high solubility, this monosaccharide does not crystallize from an aqueous solution. These functions of fructose can then be used to improve food quality (controlling microbial growth, unwanted crystallizations and improving texture) and to extend the shelf-life of different foods (Hanover \& White, 1993). However, the high hygroscopicity of fructose implies that it more readily adsorbs water from the external environment than other sugars (saccharose, for example); this fact must be taken into account during storage: an ideal packaging is needed to avoid caking or lumping problems. Fructose was used in the formulation of 'Amaretti' also for its sweetness. In fact, it is the sweetest of all natural carbohydrates. Having been assigned value of 100 to saccharose, the sweetness of fructose relative to saccharose is 117 . Moreover, fructose shows a synergy with other sweeteners present in the same formulation (for example, the relative sweetness of a 1:1 fructose/saccharose mixture is 128) (Hanover \& White, 1993). Fructose can then be used to increase the global sweetness of products without increasing the total amount of sweeteners or, in others words, the formulator could obtain a same level of sweetness while reducing the total level of sweeteners by using fructose. Bamboo fibre is a new cellulose-based insoluble ingredient derived from the fibre-rich parts of bamboo plants, with a total fibres content higher than $99 \%$ (dry basis), an high pectin and hemicellulose content, a length of about $0.25 \mathrm{~mm}$ and a density of about $120 \mathrm{~g} \mathrm{~L}^{-}$ 1; moreover, this purified fibre has a WHC (water holding capacity) of $8.7 \mathrm{~g} \mathrm{~g}^{-1}$ fibre, calculated after centrifugation, showing that it is able to retain water not only by capillary forces but by specific bonds as well (data provided by the supplier). For these reasons, the addition of bamboo fibre makes it possible to lower the $\mathrm{a}_{\mathrm{w}}$ value considerably; it can contribute beneficial textural properties also, because it is considered as a texturizing agent, improving mouth feel too. Moreover, fibres are credited to have a lot of functional properties: the shortening of intestinal transit time, the slowing down of carbohydrates sorption (controlling the glucose level in diabetic subjects), the 
control of total cholesterol level in the blood, the weight management etc., as reported in several papers (Anderson \& Chen, 1979; Bessesen, 2001; Bingham, 1987; Brown et al.,1999; De Vries, 2003; Lupton \& Turner, 2003; Kelsay,1978; Margetts et al., 1988; Miettinen, 1987; StasseWolthuis et al., 1980; Trowell, 1976; Wahlqvist, 1987). In addition to the above mentioned functional and technological properties, fructose and bamboo fibre were added to the original recipe as a result of growing consumer interest in health foods and beverages, with nutritional aspects that would make them more attractive to consumers. However, it is well evident that the supplement of these ingredients to the cookie formulation deeply affects the process conditions, especially in terms of temperature and time of baking. Therefore, the aim of the present research was to investigate the effects of the new ingredients and of baking conditions on the characteristics of finished product that are relevant to the consumer appreciation; to this purpose, the experimental investigation was carried out by a screening test, using a fractional factorial design. This kind of designs can serve as a useful and powerful tool for focusing large numbers of variables and thus reducing the number of experiments in the subsequent modelisation or optimisation phase, as highlighted by different authors (Acodedji, 2003; Araujo \& Brereton, 1996; Baardseth et al., 2005; Guha et al., 2003; Lund, 2003). Moreover, to our knowledge there is a lack of the example of practical use of the screening tests as a first preliminary stage of an experimental investigation for the development and enhancing of traditional food items.

\section{Materials and methods}

\section{The manufacturing of the 'Amaretti'}

'Amaretti' cookies were made in the Department pilot plant, using the traditional procedure (Piga et al., 2005). The typical recipe of 'Amaretti' is shown in Table 1. For the experimental design bamboo fibre and crystalline fructose [Chimab S.p.a., via C. Colombo, 34 - 35011 Campodarsego (PD) - Italy] were added in the amounts reported in Table 1. 


\section{Storage of the 'Amaretti'}

After baking, the 'Amaretti' were stored under controlled temperature-humidity conditions $(T=25$ $\pm 0.5^{\circ} \mathrm{C}$ and $R H=40 \pm 2 \%$ ) for $2 \mathrm{~h}$ before analyses.

\section{Water activity and moisture content determination}

Water activity was measured on three 'Amaretti' at a time per each run by an electronic hygrometer (LabMaster- $a_{w}$ Axair AG, Novasina, Pfäffikon, Switzerland), previously calibrated with six different standard salts of known activity (prepared by High-Purity Standards for Novasina). Gravimetric analysis was performed in triplicate to determine water content $\left(\% \mathrm{H}_{2} \mathrm{O}\right.$ on wet basis) using an oven at $130 \pm 2.0{ }^{\circ} \mathrm{C}$ for $90 \mathrm{~min}$. The measurements were performed on the internal paste of the cookies, sampling $5 \pm 0.1 \mathrm{~g}$ of product from the inner part of the almond paste filling with a laboratory spattle.

\section{Texture analysis}

Hardness was evaluated in the freshly baked 'Amaretti' using a food texture analyser (mod. Z005, Zwick Roell, Ulm, Germany). The software TESTXPERT V10.11 MASTER was used for data analysis. Textural determination was made on 15 'Amaretti' per each run by a puncturing test (Bourne, 2002). The area under the obtained curve $(\mathrm{N} \times \mathrm{mm})$ was considered as an index of global hardness of the cookie $\left(W_{t o t}\right)$. Even if a lot of textural indices can be used as dependent variables depending on the research scope, hardness was selected in this work since the best parameter able to fit with textural changes of 'Amaretti' cookies perceived by consumers during time (Farris et al., 2006).

\section{Colour measurement}

Colour analysis was carried out on freshly baked 'Amaretti' (30 replicates per each lot) using a D65 illuminant $/ 10^{\circ}$ observer reflection colorimeter (MINOLTA Chroma Meter mod. CR 210, Osaka, Japan). Cookies were placed on a white standard plate $\left(L^{*}=100\right)$ and the $C I E L * a * b *$ co-ordinates 
were simultaneously measured, even if only the $L^{*}$ parameter was considered in the experimental design.

\section{Statistical analysis}

Data were subjected to one-way ANOVA using STATGRAPHICS PLUS 4.0 software, followed by least significant difference multiple range test $(\mathrm{P} \leq 0.05)$ for comparison of the mean values. MODDE software package (MODDE 2006, version 8.0; UMETRICS AB, Umea, Sweden) was used for evaluation of raw data and regression analysis in the screening design, according to the least squares analysis technique.

\section{Experimental design}

Five quantitative controllable factors were considered for the screening test: bamboo fibre $\left(X_{l}\right)$, Fructose/Saccharose ratio $\left(X_{2}\right)$, from now on indicated as $\mathrm{F} / \mathrm{S}$, and egg white $\left(X_{3}\right)$ as ingredients; temperature $\left(X_{4}\right)$ and baking time $\left(X_{5}\right)$ as process conditions. Each of these independent variables was assessed at two equidistant levels $(-1$ and +1$)$ from the centre point $(0)$. These levels are given in Table 2. Four dependent variables (responses) were selected to represent the parameters of 'Amaretti' quality: hardness $\left(Y_{1}\right)$, moisture content $\left(Y_{2}\right)$, water activity $\left(Y_{3}\right)$ and colour $\left(Y_{4}\right)$. Taking into account the available resources, a $2^{5-1}$ fractional factorial design of resolution $\mathrm{V}$ was chosen, with 21 total runs (16 corner points and 5 centre-point replicates). This design supports an interaction model, estimable with a reduced factorial design, in which only a fraction (sixteen) of all possible corners (thirty-two) are investigated. The worksheet obtained from this design is reported in Table 3. As far as the selection of the regression model is concerned, it was assumed that $i$ mathematical functions, $\mathrm{f}_{\mathrm{z}}(z=1,2 \ldots i)$ exist for each response, $Y_{z}$, function of $l$ independent factors, $X_{\mathrm{k}}(\mathrm{k}=1,2, \ldots l)$, such that:

$$
Y_{z}=f_{z}\left(X_{1}, X_{2}, \ldots . X_{l}\right)
$$


where: $i=4$ and $l=5$. Finally, the $\mathrm{f}_{\mathrm{z}}$ function was assumed to be approximated by a polynomial equation:

$$
Y_{z}=b_{z 0}+\sum_{k=1}^{l=5} b_{z k} X_{k}+\sum_{k \neq j}^{l=5} b_{z k j} X_{k} X_{j}+\varepsilon
$$

where:

$-Y_{z}=$ dependent variable;

- $b_{z 0}=$ response value when all factors are set at medium level (centre point);

- $b_{z k}=$ linear regression coefficient;

- $b_{z k j}=$ interaction regression coefficient;

$-\varepsilon=$ residual response variation not explained by the model.

The selected regression model and the correlated data analysis provided a proper understanding concerning the correlation between each factor and different responses, by estimating the numerical values of the model terms, i.e. the regression coefficients.

\section{Results and discussion}

\section{Selection of regression model}

At first, the raw data analysis was carried out. The replicate plot (in which the measured values of a response are plotted against each run) is a useful graphical tool to compare the experimental error (noise) with the variation across all samples (effect) due to the factors setting. For hardness, moisture content and colour responses the variation in the five replicates was much smaller than the variation in the entire investigation series, i.e. the replicate error did not complicate the data analysis. As an example, replicate plot pertaining to the hardness response is shown in Fig. 1a. In this plot, any experiment appears on an isolated bar, except for the five replicates at the centre point, that are displayed on a same bar (the 17 th one). For $a_{w}$ response (Fig. 1b), on the contrary, the 
highest difference between two replicates $\left(\Delta_{17-18}=0.756-0.725=0.005\right)$ was greater than the lowest difference between two other runs of the design $\left(\Delta_{13-14}=0.712-0.708=0.004\right)$ : as we'll see shortly, this fact affected the global power of the model. All main linear and interaction coefficients were calculated for each model (Table 4); only the regression coefficients significant at $95 \%$ level were selected for developing the models. The other ones outside of this confidence level may be removed and the model refitted to the data. In this case, two interaction terms (fibre*F/S and egg white* $F / S$ ) were removed. To assess the power of fitting, and so the power of the obtained models, two main important factors were considered:

- $\quad R^{2}$, called power of fit, that represents the explained variation, i.e. the variation that can be described and modelled; it is a measure of how well the regression model fits the raw data. $R^{2}$ ranges between 0 and 1 , where 1 is for perfect models, in which predicted and observed values are equals; $R^{2}$ alone is not sufficient to asses the power of the model, because it can be easily approached to 1 by adding additional terms in the model.

- $\quad Q^{2}$, called power of prediction, is a better indicator of the power of a model; in fact, it estimates the predictive capacity that is the final objective of the model: prediction of new experiments. $Q^{2}$ ranges between 1 and $-\infty$ and, in a screening design, values of 0.5 and 0.9 are judged good and excellent, respectively. Moreover, the difference $R^{2}-Q^{2}$ should be < 0.3 .

Two further parameters usually taken into account are the model validity (a measure of model imperfection; a value $<0.25$ indicates significant lack of fit) and the reproducibility (as a measure of the accuracy of replicates; if it is below 0.5 , a large replicate error exists and a consequent poor control of the experimental procedure). Specific values of the above mentioned four model parameters are reported in Table 5 showing that two excellent, one good and one poor models (for hardness and colour, moisture content, water activity, respectively) were obtained. The negative $Q^{2}$ value for water activity is linked to the high experimental error, i.e. the replicated experiments spread too much. Statistical analysis showed that the observed differences in $a_{w}$ among 'Amaretti' 
obtained according to each designed run are not significant because they are within the experimental error, although some effect due to factors setting was observed. Probably, this is because the selected experimental region (defined by the factors setting $-1 /+1$ ) was not large enough to justify statistically different $a_{w}$ responses in its narrow range $0-1$. Because data variations concerning $a_{w}$ response were not adequately explained by the model, it can not be used for prediction purposes, but it may be useful for trend analysis. For that reason, from this step on it has no longer been taken into account. Hence, the three polynomial equations that allow modelling the relationship between factors and each response are:

- Hardness $\left(Y_{1}\right)=30.64+3.65 \mathrm{X}_{1}-3.08 \mathrm{X}_{3}+10.59 \mathrm{X}_{4}+5.14 \mathrm{X}_{5}+0.88 \mathrm{X}_{1} \mathrm{X}_{3}-0.69 \mathrm{X}_{1} \mathrm{X}_{4}+$ $+2.65 \mathrm{X}_{1} \mathrm{X}_{5}-1.04 \mathrm{X}_{2} \mathrm{X}_{4}+0.81 \mathrm{X}_{2} \mathrm{X}_{5}-2.10 \mathrm{X}_{3} \mathrm{X}_{4}-0.93 \mathrm{X}_{4} \mathrm{X}_{5}$

- $\quad$ Moisture content $\left(Y_{2}\right)=-1.92+6.51 * 10^{-3} \mathrm{X}_{2}+1.29 * 10^{-3} \mathrm{X}_{3}-4.71 * 10^{-3} \mathrm{X}_{4}-2.98 * 10^{-3} \mathrm{X}_{5}+$ $+1.41 * 10^{-3} \mathrm{X}_{1} \mathrm{X}_{4}-1.22 * 10^{-3} \mathrm{X}_{3} \mathrm{X}_{4}-1.21 * 10^{-3} \mathrm{X}_{4} \mathrm{X}_{5}$

- $\operatorname{Colour}\left(Y_{4}\right)=0.47-0.069 \mathrm{X}_{2}-0.021 \mathrm{X}_{3}-0.263 \mathrm{X}_{4}-0.053 \mathrm{X}_{5}+0.025 \mathrm{X}_{1} \mathrm{X}_{3}-0.035 \mathrm{X}_{2} \mathrm{X}_{4}+$ $+0.029 \mathrm{X}_{3} \mathrm{X}_{5}-0.038 \mathrm{X}_{4} \mathrm{X}_{5}$

\section{Analysis of variance}

Once the models were selected, analysis of variance was carried out, to assess the global validity of the models. In particular (Table 6), the first $F$-test confirmed that all three models adequately represented the data for hardness, moisture content and colour. This is because the variance explained by each model (Mean Squares regression) was significantly larger than the amount of unexplained variance, i.e. that could not be described by the model (Mean Squares residual). In the second test (lack of fit test), the unexplainable variance was considered as a sum of two parts: one (Mean Squares model error) due to the implied imperfections of the models, the other one (Mean Squares replicate error) linked to the replicate error (variation in the replicated experiments). The lack of fit test showed that model error and replicate error were small and had similar size, that is there was 
no lack of fit: the models were sufficiently accurate for predicting each corresponding response. Moreover, there were no outliers to the regression.

\section{Evaluation of the model}

The obtained models achieved the goal of this study, i.e. a better understanding of the most important factors influencing each response. Figure 2 shows the influence of each factor (as main linear and as interaction effect) on hardness, moisture content and colour. As far as hardness is concerned, it is possible to observe that temperature, time and fibre influenced positively this response, while increasing the amount of egg white, the hardness decreased. Moreover, it is important to stress the statistically significant interaction effect Fibre*Time (Fig. 3): with increase of fibre, the hardness of the 'Amaretti' increased. However, the influence of fibre was greater when baking time was high. So, the effect of fibre depended on baking time. Moisture content response was significantly affected by three main factors: F/S ratio that acted positively, temperature and time that acted negatively. Finally, colour was mainly influenced by temperature. Through the increase in this parameter, $L^{*}$ value decreased and so the browness of 'Amaretti' increased. F/S ratio and baking time played a less important role, but not negligible. The response surface contour plots are useful tools to interpret the relationship between main factors and responses and to indicate the direction to modify the variables to obtain the desired results. These plots were obtained varying two variables within the experimental range, while the other three were kept constant at the optimum level. Some selected response surface plots are presented in Figs 4-8. The relationship of hardness with time and temperature is shown in Fig. 4. Both factors influenced hardness in the same direction, even if the unit change in time produced an inferior change in response than temperature. Moreover, it is possible to observe that intermediate-high response values can be obtained for high baking time and medium temperature in the investigated factors range. Figure 5 shows the influence of fibre and time factors on hardness. For the lowest baking time values (i.e. $18 \mathrm{~min}$ ), increasing fibre did not lead to any increase of hardness response. The influence of fibre on hardness can be 
considered appreciable only for a baking time higher than the medium level (20 min). In other words, the effect of fibre is strictly baking-time dependent. The influence of the two most important factors affecting moisture content response is reported in Fig. 6. Here it is possible to observe that temperature and F/S ratio behaved on the same response in a diametrically opposed way: increasing $\mathrm{F} / \mathrm{S}$ ratio moisture content increased, while increasing baking temperature moisture content decreased. So, intermediate moisture content values can be obtained by an intermediate setting of these two factors. Figure 7 explains the effect of temperature and F/S ratio on colour. The increase in both caused a decrease in $L^{*}$ parameter, even if the effect of temperature was greater than that of F/S. Finally, the effect of fibre and egg white on colour was considered (Fig. 8). In this case, a typical example of an interaction effect is given: for the lowest levels of egg white, the increase in baking time led to a higher browness of 'Amaretti'. Increasing the amount of egg white, reversed the result and increasing baking time from 18 to $22 \mathrm{~min}$, the cookies showed a little less dark colour.

\section{Conclusions}

Design Of Experiment (DOE) technique was successfully applied to evaluate the effect of different factors on the most important quality parameters of 'Amaretti' cookies. Three of the four models developed in this research resulted appropriated when simultaneous responses (hardness, moisture content, water activity and colour) were considered. Starting from these models, it was possible to achieve a deeper knowledge as far as the influence of each factor on responses is concerned. Hardness was affected mainly by oven temperature, baking time and fibre; F/S ratio, oven temperature and baking time influenced both moisture content and colour. Egg white influenced all responses, both as main effect and as interaction term, but its effect was lesser than the other factors. Its greater effect could arise as quadratic term. From these results some main consideration can be inferred about the more favourable formula and process conditions of 'Amaretti'. At first, a 
high amount of fructose should not be used in order to avoid an excessive and unacceptable brown colour. A similar effect could arise from the highest temperature level. Secondly, typical values of moisture content of the soft almond-based filling seems to be guaranteed by the combined effect of a medium amount of egg white and the lowest F/S ratio, even though medium temperatures are set. Finally, improvement in mouth feel is also given by the fibre that could be used at fairly high amount. However, the interaction effect between fibre and baking time must be considered. In this sense, baking time should be set around its medium level to avoid hard cookies. In any case, it is important to emphasize that the new ingredients added to the original recipe appear to play an important role on overall quality of the cookies; for that reason, they can be rightly used to enhance this traditional Italian product. So, in a further research it will be important to gain a better understanding of the relationship between factors and responses. To achieve this aim, a quadratic model is needed, to take into consideration all quadratic effects too. This will be possible through the response surface modelling technique that, finally, can be used to pinpoint the best combination of factors (i.e. the best recipe for 'Amaretti').

Acnowledgements We thank Lorenza Broccardo (UMETRICS, S-IN Soluzioni Informatiche, Vicenza, Italy) for the useful advice in the design development. We acknowledge Laura Introzzi, who contributed to make this work during her thesis project. 


\section{References}

(1) Acodedji, E.C. (2003). Projective properties of screening designs in economic construction of modelisation or optimisation designs. Chemometrics and Intelligent Laboratory Systems, 67, 21-28.

(2) Anderson, J.W. \& Chen, W.J. (1979). Plant fibre. Carbohydrate and lipid metabolism. American Journal of Clinical Nutrition, 32, 346-363.

(3) Araujo, P.W. \& Brereton, R.G. (1996). Experimental designs I. Screening. Trends in Analytical Chemistry, 15, 26-31.

(4) Baardseth, P., Bjerke, F., Aaby, K. \& Mielnik, M. (2005). A screening experiment to identify factors causing rancidity during meat loaf production. European Food Research and Technology 221, 653-661.

(5) Bessesen, D.H. (2001). The role of carbohydrates in insulin resistance. Journal of Nutrition, 131, 2782S-2786S.

(6) Bingham, S. (1987). Definitions and intakes of dietary fibre. American Journal of Clinical Nutrition, 45, 1226-1231.

(7) Bourne, M.C. (2002). Food texture and viscosity: concepts and measurement (International Series ed.), $2^{\text {nd }}$ edn. Pp. 113-126. Academic Press, New York/London.

(8) Brown, L., Rosner, B., Willet, W.W. \& Sacks, F.M. (1999). Dietary fibre: a meta-analysis. American Journal of Clinical Nutrition, 69, 30-42.

(9) De Vries, J. (2003). On defining dietary fibre. Proceedings of the Nutrition Society, 62: 37-43.

(10) Farris, S., Limbo, S., \& Piergiovanni, L. (2006).Effect of two different humectant ingredients on quality of 'Amaretti' cookies. In Proceedings of Shelf Life International Meeting, Catania, I. Special Issue of Italian Journal of Food Science (edited by G. Muratore and F. Licciardello). Pp. 247-254. Pinerolo, Italy: Chiriotti Press.

(11) Guha, M., Ali, S.Z. \& Bhattacharya, S. (2003). Screening of variables for extrusion of rice flour employing a Plackett-Burman design. Journal of Food Engineering, 57, 135-144.

(12) Guillard, N., Broyart, B., Guilbert, S., \& Gontard, N. (2003a). Preventing moisture transfer in a composite food using edible films: experimental and mathematical study. Journal of Food Science, 68, 2267-2277.

(13) Guillard, N., Broyart, B., Guilbert, S., \& Gontard, N. (2003b). Modelling of moisture transfer in a composite food: dynamic water properties in an intermediate $\mathrm{a}_{\mathrm{w}}$ porous product in contactwith high $\mathrm{a}_{\mathrm{w}}$ filling. Transaction of the Institution of Chemical Engineers, 81, 1090-1098.

(14) Guilbért, S. (2002). Additifs et Agents Depresseur de l'Activitè de l'Eau. In : Additifs et Auxiliares de Fabrication dans les Industries Agroalimentaires (edited by J.L. Multon), $3^{\text {rd }}$ edn. Pp. 237-265. Paris: TEC \& DOC Press.

(15) Hanover, L.M. \& White, J.S. (1993). Manufacturing, composition and applications of fructose. American Journal of Clinical Nutrition, 58 (suppl.), 724S-732S.

(16) Kelsay, J.L. (1978). A review of research on effects of fibre intake on man. American Journal of Clinical Nutrition, 31, 142-159. 
(17) Labuza, T.P., \& Hyman, C.R. (1998). Moisture migration and control in multi-domain foods. Trends in Food Science \& Technology, 9, 47-55.

(18) Lund, D. (2003). Predicting the impact of food processing on food constituents. Journal of Food Engineering, 56, 113-117.

(19) Lupton, J.R. \& Turner, N.D. (2003). Dietary fibre and coronary disease: does the evidence support an association? Current Atherosclerosis Reports, 5, 500-505.

(20) Manzocco, L. \& Nicoli, M.C. (2002). Food design: from the methodological approach to the case study of low-calorie syrups. Trends in Food Science \& Technology, 13, 420-427.

(21) Miettinen, T.A. (1987). Dietary fibre and lipids. American Journal of Clinical Nutrition, 45, 1237-1242.

(22) Margetts, B.M., Beilin, L.J., Armstrong, B.K. \& Vandongen, R. (1988). Vegetarian diet in mild hypertension: effects of fat and fibre. American Journal of Clinical Nutrition, 48, 801-805.

(23) Piga, A., Catzeddu, P., Farris, S., Roggio, T., Sanguinetti, A. \& Scano, E. (2005) . Texture evolution of 'Amaretti' cookies during storage. European Food Research Technology, 221, 387-391.

(24) Ramos-Cabrer, P., Van Duynhoven, J.P.M., Timmer, H., \& Nicolay, K. (2006). Monitoring of moisture redistribution in multicomponent food systems by use of magnetic resonance imaging. Journal of Agricultural and Food Chemistry, 54, 672677.

(25) Roca E., Guillard, V., Guilbert, S., \& Gontard, N. (2006). Misture migration in a cereal composite food at high water activity: effect of initial porosity and fat content. Journal of Cereal Science, 43, 144-151.

(26) Stasse-Wolthuis, M., Albers, H.F.F., van Jeveren, J.G.C., de Jong, J.W., Hautvast, J.G.A.J., Hermus, R.J.J., Katan, M.B., Brydon, W.G. \& Eastwood, M.A. (1980) . Influence of dietary fibre from vegetables and fruits, bran or citrus pectin on serum lipids, fecal lipids and colonic function. American Journal of Clinical Nutrition, 33, 1745-1756.

(27) Trichopoulou, A., Vasilopoulou, E., Georga, K., Soukara, S. \& Dilis, V. (2006). Traditional foods: why and how to sustain them. Trends in Food Science and Technology, 17, 498-504.

(28) Trowell, H. (1976). Definition of dietary fibre and hypoteses that it is a protective factor in certain diseases. American Journal of Clinical Nutrition, 29, 417-427.

(29) Wahlqvist, M.L. (1987). Dietary fibre and carbohydrate metabolism. American Journal of Clinical Nutrition, 48, 801-805. 


\section{Legends to Figures.}

Figure 1a Plot of replications for hardness response.

Figure 1b Plot of replications for $a_{w}$ response.

Figure 2 Coefficient overview plot for all three responses.

Figure 3 Interaction plot for Fibre*Time; response: hardness.

Figure 4 Response contour plot for Time*Temperature; response: hardness.

Fibre: 30 g. F/S: 0.1. Egg white: 265 g.

Figure 5 Response contour plot for Fibre*Time; response: hardness.

Temperature: $195^{\circ} \mathrm{C} . \mathrm{F} / \mathrm{S}: 0.1$. Egg white: $258 \mathrm{~g}$.

Figure 6 Response contour plot for Temperature*F/S; response: moisture content.

Fibre: 30 g. Egg white: 265 g. Time: 20 min.

Figure 7 Response contour plot for Temperature*F/S; response: colour.

Fibre: 30 g. Egg white: 265 g. Time: $20 \mathrm{~min}$.

Figure 8 Response contour plot for Time*Egg white; response: colour.

Fibre: 30 g. F/S: 0.1 . Temperature: $165^{\circ} \mathrm{C}$. 